Carnets de géographes

GÉOGRAPHES.

$3 \mid 2011$

Les géographies des enfants et des jeunes

\title{
Sociabilité numérique et mobilité des adolescents : des favelas de Rio aux banlieues parisiennes
}

Helene Petry

\section{(2) OpenEdition}

Journals

Édition électronique

URL : http://journals.openedition.org/cdg/2250

DOI : $10.4000 /$ cdg. 2250

ISSN : 2107-7266

Éditeur

UMR 245 - CESSMA

Référence électronique

Helene Petry, "Sociabilité numérique et mobilité des adolescents : des favelas de Rio aux banlieues parisiennes », Carnets de géographes [En ligne], 3 | 2011, mis en ligne le 01 décembre 2011, consulté le 07 mai 2019. URL : http://journals.openedition.org/cdg/2250 ; DOI : 10.4000/cdg.2250

La revue Carnets de géographes est mise à disposition selon les termes de la Licence Creative Commons Attribution - Pas d'Utilisation Commerciale - Pas de Modification 4.0 International. 


\section{SOCIABILITE NUMERIQUE ET MOBILITE DES ADOLESCENTS :}

Des favelas de Rio aux banlieues parisiennes

HELENE PETRY

Institut d'Etudes Politiques de Paris

Sociologie

helene.petry@gmail.com

\section{Résumé}

À partir d'une enquête de terrain menee dans des etablissements secondaires publics à Rio de Janeiro et en region parisienne, cet article montre que la forte connectivite numerique des adolescents (telephone portable et Internet) favorise leur mobilite spatiale et leur sociabilite. En revanche, elle les prend egalement dans un devoir moral de "joignabilite " qui se joue surtout face à la famille dans le groupe carioca et face aux amis du même âge dans le groupe francilien 
La tendance des TIC traditionnelles comme la television à privatiser les loisirs a ete mise en avant comme defavorable aux interactions sociales (Putnam, 2000). Les NTIC à l'inverse encouragent les interactions sociales en entrelaçant loisir et communication (Smoreda, 2007). À partir d'une enquête de terrain menee dans des etablissements secondaires publics à Rio de Janeiro et en region parisienne, cet article $^{2}$ montre que la forte connectivite numerique des adolescents (telephone portable et Internet) favorise leur mobilite spatiale en augmentant leurs pratiques de sociabilite ${ }^{3}$ aussi bien numeriques qu'en copresence.

La sociabilite a une importante dimension spatiale dans la mesure où elle est façonnee par les caracteristiques des espaces où elle peut se derouler (Florida, 2003; Jacobs, 1989; Joseph, 1998). La multiplication des communications numeriques affecte donc la façon d'être ensemble, creant de nouveaux espaces numeriques de sociabilite qui interpellent les sciences sociales. Selon Boris Beaude :

(...) comme cette spatialité est inédite, il faut prendre la peine de comprendre en quoi elle est singulière, comprendre en quoi, potentiellement, cet espace peut changer la société dans son ensemble, si on considère que la société, c'est du lien social et que le lien social dépend du contact, donc de ses lieux. (...) Si on a de nouveaux lieux, il y a de nouvelles façons d'être ensemble. (Beaude, 2011)

Une abondante litterature affirme que la generation Internet pense, apprend, travaille et interagit de manière significativement differente des generations precedentes en raison de son usage fort et precoce des NTIC, sans toutefois degager de consensus sur la nature de ces changements (Bauerlein, 2008; Howe \& Strauss, 1991; Lardellier \& Bryon-Portet, 2010; PewResearchCenter, 2010; Prensky, 2001; Tapscott, 2008; Twenge, 2006). Alors que plusieurs travaux empiriques sont venus relativiser les competences numeriques de cette generation (Hargittai, 2010; Selwyn, 2009; Zimic, 2010), le constat d'une evolution majeure de ses modes de sociabilite demeure inconteste. Celles-ci ont donc fait l'objet de nombreuses recherches dans divers pays. En France, où le sujet interesse de plus en plus les sociologues, les etudes mettent en avant le rôle des sociabilites numeriques dans le renforcement et l'autonomisation du groupe de pairs (Delaunay-Teterel, 2010; Jarrigeon \& Menrath, 2010; Metton, 2010; Pasquier, 2005). Au Bresil, où ces sociabilites sont analysees principalement dans les champs de la psychologie et de la communication, elles sont davantage abordees à travers les questions de l'identite et des communautes virtuelles (Correa, 2006; Nicolaci-da-Costa, 2005; Passarelli Hamann, 2004; Recuero, 2006; Rezende Sales \& Alves Paraiso, 2010; Silva Pereira, 2007). Ces travaux font saillir des alterites et des unites plus pertinentes que les differences nationales dans le domaine des pratiques numeriques, au premier rang desquelles les differences entre milieu urbain et milieu rural, mais surtout les differences d'âge et de generation (Beck \& Beck-Gernsheim, 2008; Edmunds \& Turner, 2005). Toutefois, l'etude qualitative des sociabilites numeriques fait apparaître

\footnotetext{
1 NTIC: Nouvelles Technologies d'Information et de Communication. Également appelées TNIC: Technologies Numériques d'Information et de Communication.

2 Je tiens à remercier les deux rapporteurs anonymes ainsi que le Comité de rédaction des Carnets de Géographes pour leurs commentaires et suggestions m'ayant permis d'améliorer une version précédente de cet article.

3 Comprises comme l'ensemble des pratiques volontaires d'interactions entre individus, qui créent et entretiennent des relations sociales temporaires ou durables. Pour une mise en perspective théorique du concept de sociabilité, voir l'article de Rivière (Rivière, 2004).
} 
comment les usages de ces moyens de communication repondent à des normes sociales qui varient selon les contextes culturels.

Neanmoins, un risque majeur de l'etude de ces nouvelles façons d'être ensemble est de se concentrer uniquement sur la dimension numerique en l'isolant des activites hors ligne alors qu'une part importante des pratiques numeriques et physiques de sociabilite sont interdependantes. La principale question de recherche qui guide cet article consiste donc à comprendre l'imbrication de l'espace numerique et de l'espace physique de sociabilite des lyceens de la peripherie de grandes metropoles à travers l'etude de leur mobilite. Dans le but de faire apparaître des dynamiques locales mais aussi globales, l'etude s'est interessee à des lyceens d'une metropole du Nord, Paris, et du Sud, Rio de Janeiro aux caracteristiques spatiales et sociales très distinctes. L'article presente tout $d$ 'abord les terrains et les donnees recueillies, mettant en avant les specificites des favelas et des banlieues etudiees dans leurs contextes urbain et demographiques respectifs, ainsi que les caracteristiques communes aux deux groupes motivant le choix $d^{\prime}$ 'une etude transnationale ${ }^{4}$. La deuxième partie presente les resultats concernant la sociabilite centree sur le groupe de pairs dont les liens internes et l'autonomie par rapport aux adultes sont renforces par les communications numeriques, particulièrement dans le groupe francilien. La troisième partie developpe les resultats sur les communications numeriques avec la famille, qui visent à concilier solidarite familiale et mobilite spatiale, particulièrement dans le groupe carioca.

\section{Les lycéens de banlieues populaires parisiennes et de favelas cariocas : présentation de la génération Internet dans des contextes de vie contrastés}

Les groupes etudies s'inscrivent dans des contextes locaux contrastes (a), mais appartiennent à une generation internet globale (b) dont les pratiques de sociabilite demeurent marquees par les cultures nationales (c).

\section{Deux contextes socio-urbains très différents}

Deux differences principales doivent être soulignee concernant les banlieues populaires franciliennes et les favelas cariocas. La première concerne la mesure de la pauvrete et des inegalites sociales dans les deux pays. Alors que la France presente un indice de $\mathrm{Gini}^{5}$ de 0,3, le Bresil a une des distributions de revenus les plus inegalitaires au monde avec un Gini de 0,6 , et une population pauvre beaucoup plus nombreuse et precaire que dans le cas français $^{6}$. Les figures 1 et 2 ci-dessous presentent quelques repères sur le profil social moyen

\footnotetext{
${ }^{4}$ L'étude transnationale se distingue de la comparaison internationale du fait qu'elle ne vise pas la comparaison terme à terme qui fait saillir des différences et spécificités nationales, mais l'étude d'un même phénomène de pertinence transnationale ou globale à travers des enquêtes dans des localités qui dépassent les frontières nationales.

${ }^{5}$ L'indice Gini est une mesure des inégalités de revenus allant de 0 (égalité parfaite) à 1 (inégalité totale).

${ }^{6}$ À titre indicatif, le salaire mensuel minimum légal au moment de l'enquête est de $1200 €$ en France, et de $415 \mathrm{R} \$(145 €)$ au Brésil, pour un coût de la vie à Rio de Janeiro comparable à celui d'une ville moyenne française. Par ailleurs, il n'existe pas d'indemnité de chômage au Brésil, ni d'habitat social, mais la bolsa familia, qui octroie une aide de 20 à $200 \mathrm{R} \$(7$ à $70 €$ ) pour les familles dont les revenus mensuels sont inférieurs à $200 \mathrm{R} \$$.
} 
des habitants des quartiers où resident la majorite des elèves enquêtes, mis en perspective par rapport aux moyennes nationales de chaque pays.

Figure 1 : Indicateurs sociaux pour les quartiers étudiés en région parisienne (2006)

\begin{tabular}{|l|r|r|r|r|}
\hline & $\begin{array}{c}\text { Revenu brut } \\
\text { mensuel individuel } \\
\text { moyen }\end{array}$ & $\begin{array}{c}\text { Taux de propriété } \\
\text { du logement }\end{array}$ & $\begin{array}{c}\text { Taux d'habitat } \\
\text { social }\end{array}$ & $\begin{array}{c}\text { Proportion } \\
\text { d'étrangers dans } \\
\text { la population }\end{array}$ \\
\hline Quartiers étudiés* & $1020 €(2)$ & $28 \%(2)$ & $38 \%(2)$ & $21 \%(4)(5)$ \\
\hline France & $1550 €(1)$ & $57 \%(1)$ & $17 \%(1)$ & $5,7 \%(3)$ \\
\hline
\end{tabular}

Sources: 1) INSEE. 2) Institut d'Amenagement et d'Urbanisme (IAU) île-de-France. 3) Infos migrations, Ministère de I'immigration, de l'integration, de l'identite nationale et du developpement solidaire. 4) AEDI (2008). 5) PCF (2008).

* Moyenne des donnees pour les quatre communes regroupant la majorite des elèves des deux etablissements etudies.

Figure 2 : Indicateurs sociaux pour les quartiers étudiés à Rio de Janeiro (2008)

\begin{tabular}{|l|l|l|l|}
\hline & \multicolumn{1}{|c|}{$\begin{array}{c}\text { Élite } \\
\text { (classes A et B)** }\end{array}$} & \multicolumn{1}{|c|}{$\begin{array}{c}\text { Classe moyenne } \\
\text { (classe C)** }\end{array}$} & $\begin{array}{c}\text { Pauvres et indigents } \\
\text { (Classes D et E)** }\end{array}$ \\
\hline Quartiers étudiés* (1) & $<1 \%$ & $30 \%$ & $70 \%$ \\
\hline Brésil (2) & $15,5 \%$ & $52 \%$ & $32,5 \%$ \\
\hline
\end{tabular}

Sources: 1) IBASE. 2) Fundação Getulio Vargas/FGV citee dans (Epoca, 2008).

* Moyenne des donnees pour les deux quartiers etudies.

** Les classes sociales bresiliennes sont definies en fonction du revenu brut mensuel du foyer comme suit : Classe $A=p l u s$ de $R \$ 9181$; Classe $B=$ de $R \$ 4591$ à $R \$ 9180$; Classe $C=$ de $R \$ 1065$ à $R \$ 4590$; Classe $D=$ de $R \$ 768$ à $R \$ 1064$; Classe $E=$ moins de $R \$ 768$. Source: Fundação Getulio Vargas (FGV) citee dans (Epoca, 2008).

En vue d'eviter les " effets etablissement ", et donc pour avoir une meilleure generalisabilite de l'analyse, l'enquête s'est deroulee dans deux etablissements dans chaque metropole. Les etablissements franciliens sont situes dans des communes de la "petite couronne " ou proche banlieue parisienne; je les appellerai lycee Paris Nord et lycee Paris Sud. Le lycee Paris Nord se trouve à proximite d'une station de metro dans un quartier qualifie de " glauque " par les elèves en raison des voies rapides qui le traversent. Le lycee Paris Sud est moins bien desservi en transports en commun - à plus de vingt minutes à pied d'une station de metro - mais s'etend sur un large campus agreable et arbore. Les deux etablissements accueillent en grande majorite des elèves de la même commune et la commune la plus proche, qui ont moins de $20 \mathrm{mn}$ de trajet domicile-ecole.

Les deux etablissements cariocas sont des colégios estaduais c'est-à-dire des etablissements publics, frequentes par les enfants des familles n'ayant pas les moyens de payer un lycee prive, choix privilegie des classes moyennes et superieures. Je les appellerai lycee Rio Sud et lycee Rio Ouest. Le lycee Rio Sud se situe dans un quartier favorise où n'habite quasiment aucun elève, à l'exception de quelques uns dont les parents sont gardiens d'immeuble. La grande majorite des elèves de cet etablissement resident dans les deux favelas les plus proches, situees à 10 ou 20 minutes d'autobus. Le lycee Rio Ouest est situe au sein d'une grande favela d'où proviennent presque tous les elèves, excepte une minorite vivant dans des favelas limitrophes. Les donnees ont ete recueillies entre juillet 2008 et janvier 2009 auprès d'elèves de 15 à 18 ans à travers 600 questionnaires (294 à Rio et 306 en region 
parisienne) portant sur les equipements personnels et du foyer en TIC et les activites numeriques, completes par soixante entretiens individuels semi diriges (trente dans chaque pays) pour approfondir les questions de sociabilite, loisirs, et mobilite.

La figure 3 montre le profil socio-educatif [SocEdu] des familles des elèves enquêtes elabore à partir du niveau d'etudes du ou des parent(s) et de leur profession. Les resultats revèlent qu'aucun elève du groupe carioca n'appartient à un foyer à profil socio-educatif eleve ${ }^{7}$, et seulement $7 \%$ des elèves du groupe francilien sont dans ce cas. Le profil moyen ${ }^{8}$ est plus frequent dans le groupe francilien (32\%) que carioca (16\%), et dans les deux groupes, la majorite des foyers des enquêtes correspondent à un profil socio-educatif faible ${ }^{9}$, avec $61 \%$ pour le groupe francilien et $84 \%$ dans le groupe carioca.

Figure 3 : Profil socio-éducatif des foyers enquêtés

\begin{tabular}{|l|c|c|c|c|c|c|c|}
\hline & \multicolumn{2}{|c|}{ SocEdu Bas } & \multicolumn{2}{c|}{ SocEdu Moyen } & \multicolumn{2}{c|}{ SocEdu Haut } & \multicolumn{2}{c|}{ Total } \\
\hline & $n$ & $\%$ & $n$ & $\%$ & $n$ & $\%$ & \\
\hline Groupe carioca & 246 & 84 & 48 & 16 &. &. & 294 \\
\hline Groupe francilien & 184 & 61 & 95 & 32 & 21 & 7 & 300 (+6na) \\
\hline
\end{tabular}

La deuxième principale difference à souligner concernant les deux groupes etudies concerne les configurations urbaines de Paris et Rio. Dans le cas parisien, malgre la diversite sociale entre les communes de banlieue, il existe une delimitation à la fois physique et symbolique entre Paris intra-muros, le centre de l'agglomeration, et sa banlieue "hors les murs ». En revanche à Rio les morros (mornes) où se situent la plupart des favelas sont imbriques dans la ville et souvent proches des quartiers favorises, mais s'en differencient par des constructions informelles et surtout par le niveau de revenu des habitants (Valladares, 2006). Dans cette logique, la nette centralisation du reseau de metro parisien encourage les virees sur Paris des lyceens de la proche banlieue, tandis qu'à l'inverse, l'etalement de la ville de Rio, ainsi que la duree, l'insecurite, l'inconfort et souvent le coût des deplacements en bus ${ }^{10}$ y favorisent davantage le developpement de nouvelles centralites, comme autour du centre commercial Barra Shopping dans le quartier recent de Barra da Tijuca, sur le littoral ouest de Rio. Fernanda, par exemple, a renonce à suivre les samedis un stage d'informatique pour lequel son lycee lui donnait droit à une reduction, à cause des conditions de transports :

Não tinha curso mais perto ?

Por aqui não, não tão bom quanto aquele. (...). Estava fazendo, aí começou a vir o horário, pra mim era muito puxado, chegava em casa morta. O ônibus que eu pegava

\footnotetext{
7 Diplôme d'enseignement supérieur et occupation associée aux catégories 1, 2 et 3 de l'International Standart Classification of Occupations (cadres, hauts fonctionnaires, professions libérales, ingénieurs et enseignants).

8 Éducation secondaire et catégories 4, 5, et 6 de l'ISCO (employés administratifs, commerciaux, et agroindustriels qualifiés).

${ }^{9}$ Non scolarisés, ou uniquement en cycle primaire, ou un seul parent au niveau collège, et les catégories 7 , 8 et 9 de l'ISCO (petits artisans et commerçants et main d'œuvre non qualifiée des secteurs formel et informel).

${ }^{10}$ Le métro de Rio ne dessert pas les quartiers étudiés.
} 
era cheiíssimo. Então melhor eu parar. Vamos aos sábados, imagina, quatro passagens (...) duas pra ir e duas pra voltar. Impossível, ia gastar o preço do curso praticamente. Não dava. ${ }^{11}$ [Fernanda, 18 ans, lycée Rio Ouest]

En outre Paris fait figure de pôle attractif pour se promener entre amis puisque toutes les rues ont des trottoirs praticables et de nombreux quartiers sont commerçants et animes, et les elèves des deux etablissements quittent volontiers leur quartier où " il n'y a rien à faire » pour aller se promener dans Paris. À l'inverse pour le groupe carioca, dont les elèves du lycee Rio Sud ne sont pas plus distants du centre ville que les elèves parisiens, la " vie de rue » de la favela est plus riche et represente le contexte privilegie pour se retrouver entre amis - avec la plage - comme en temoigne Fabiano qui vit depuis quelques annees dans l'appartement de son beau-père dans un quartier recent de classe moyenne, mais passe tout son temps chez des cousins et amis de la Zona Sul et ses favelas :

Você gosta de morar lá ?

Não. Não tenho muitas amizades lá. (...). Só vou em casa, durmo, final de semana fico por aqui mesmo.

E não da para fazer amizades lá?

Não, todo mundo fica em casa, sei lá... É que não tem uma pracinha assim pra conversar...

Você gostaria de se mudar de lá?

Com certeza. (...) É muito chato, posso estar em casa e não ter nada pra fazer. Sair na rua, não conheço ninguém. Aqui saio na rua, encontro amigos, converso. ${ }^{12}$ [Fabiano, 18 ans, lycée Sud Rio]

Cependant, ces nettes differences geographiques et sociales sont contrebalancees par certaines caracteristiques communes aux deux groupes qui permettent de les inclure dans le cadre d'une etude transnationale sur les sociabilites numeriques.

${ }^{11}$ Il n'y avait pas de stage plus près?

Par ici non. Pas aussi bon (...). J'y allais, mais ça a commencé avec l'horaire, pour moi c'était trop, je rentrais à la maison complètement morte. Le bus que je prenais était bondé. Il valait mieux que j'arrête. C'est le samedi, imagine, quatre billets, deux à l'aller et deux au retour. Impossible, j'aurais dépensé le prix du stage, quasiment.

NB : Un billet de bus coute à l'époque 2,35 reais, soit environ $1 €$.

${ }^{12}$ Tu aimes bien habiter là-bas?

Non. J'ai pas trop d'amis là-bas. (...). Je rentre juste chez moi, je dors, le week-end je reste plus par ici.

Tu ne peux pas te faire des amis là-bas?

Non, chacun reste chez soi, je sais pas... C'est qu'il n'y a pas de petite place pour discuter...

Tu aimerais déménager?

Ça c'est sûr. (...) C'est chiant, je reste chez moi, y'a rien à faire. Si je sors dans la rue, je ne connais personne. Ici je sors, je rencontre des amis, je discute. 


\section{Une génération connectée de la périphérie des grandes métropoles qui se prête à l'étude transnationale $^{13}$}

La grande difference de ressources economiques qui existe entre les foyers à bas revenus des deux regions etudiees est tout d'abord attenuee dans les groupes etudies en raison du choix du niveau lycee. En effet, bien que le taux de scolarisation des 15-17 ans soit proche de 90\% dans l'État de Rio de Janeiro, seuls $44 \%$ d'une classe d'âge atteignent le niveau lycee (MEC, 2009). Par consequent, et etant donnee la forte correlation entre le nombre d'annees $d^{\prime}$ 'etudes des individus et le niveau economique de leur foyer d'origine, on peut estimer que la moitie la plus pauvre des adolescents cariocas n'accède pas au lycee à l'heure actuelle. En outre, le choix de lycees generaux, etablissements moins segreges que ceux du cycle precedent - collèges en France, education basique au Bresil - et que les lycees techniques ou professionnels pour la France, participe egalement à ne pas concentrer uniquement les populations les plus pauvres mais un ensemble de milieux populaires et de "petitsmoyens " (Cartier, Coutant, Masclet, \& Siblot, 2008).

Deuxièmement, malgre le fait que les groupes etudies appartiennent en majorite aux milieux consideres defavorises dans leurs pays respectifs, on observe que les taux d'equipement numerique y sont superieurs aux moyennes nationales (figure 4). On sait que les foyers des adolescents sont les plus equipes en termes de technologies numeriques dans la majorite des pays, et ce independamment des revenus du foyer (Dupuy, 2007; TNS/Sofres, 2009). Ainsi, en etant à la fois adolescents et habitants d'une grande metropole, les enquêtes cumulent les deux variables les plus favorables à la connectivite numerique (Dupuy, op. cit.). La figure 5 revèle que cet equipement ne concerne pas uniquement le telephone portable et Internet, mais une multitude d'appareils tels que le lecteur MP3, l'appareil photo numerique ou la console de jeu. Les taux d'equipement sont, comme on pouvait s'y attendre, systematiquement moins eleves dans le groupe carioca que dans le francilien, neanmoins tous les equipements possedes par plus des deux tiers des Franciliens le sont egalement par plus des deux tiers des Cariocas, à l'exception de la webcam et de l'ordinateur portable. Dans le domaine de l'equipement en tous cas, on peut donc dire que les adolescents etudies correspondent à la description de la generation connectee globale qui a emergee dans les etudes de sociologie et de marketing au cours des annees 2000 (Beck \& Beck-Gernsheim, 2008; Kjeldgaard \& Askegaard, 2006; Universal McCann, 2007).

\footnotetext{
${ }^{13}$ Les études transnationales s'inscrivent dans une critique du nationalisme méthodologique des sciences sociales (Beck, 2007; Ianni, 1998; Urry, 2000), et sont donc à distinguer des comparaisons internationales (Hassenteufel, 2005; Sassen, 2006), qui elles reposent sur le paradigme de la société nationale.
} 
Figure 4 : Taux d'équipement : téléphone portable et accès Internet à domicile (2009)

\begin{tabular}{|l|c|c|c|c|c|c|}
\hline & $\begin{array}{c}\text { Rio: } \\
\text { groupe } \\
\text { enquêté }\end{array}$ & $\begin{array}{c}\text { Brésil: } \\
\text { adolescents }\end{array}$ & $\begin{array}{c}\text { Brésil: } \\
\text { total }\end{array}$ & $\begin{array}{c}\text { Paris: } \\
\text { groupe } \\
\text { enquêté }\end{array}$ & $\begin{array}{c}\text { France: } \\
\text { adolescents }\end{array}$ & $\begin{array}{c}\text { France: } \\
\text { total }\end{array}$ \\
\hline $\begin{array}{l}\text { Téléphone } \\
\text { portable }\end{array}$ & $88 \%$ & $56 \%(2)$ & $56 \%(1)$ & $94 \%$ & $95 \%(5)$ & $82 \%(4)$ \\
\hline $\begin{array}{l}\text { Connection } \\
\text { Internet à } \\
\text { domicile }\end{array}$ & $71 \%$ & NA & $20 \%(1)$ & $96 \%$ & $96 \%(5)$ & $65 \%(3)$ \\
\hline
\end{tabular}

Sources: 1) TIC domicílios 2008. 2) PNAD 2008. 3) (Livingstone \& Haddon, 2009). 4) (CREDOC, 2009). 5) (TNS/Sofres, 2009)

Figure 5 : Equipement numérique des groupes enquêtés (en \%)

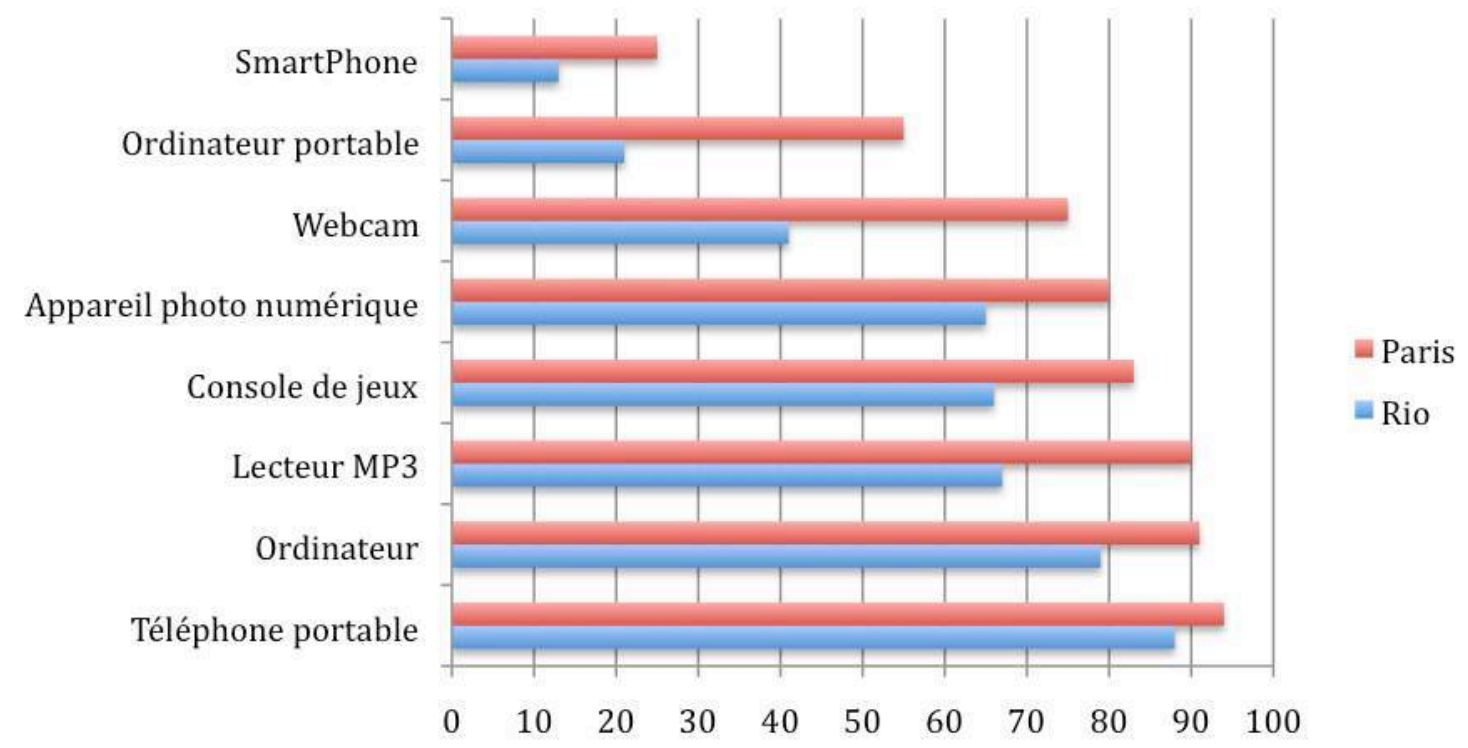

\section{Des pratiques de communication qui varient selon les contextes culturels}

Les lyceens cariocas et franciliens ont un fort taux d'usage des NTIC, avec des pratiques et des frequences d'usages comparables en ce qui concerne Internet (reseaux sociaux, recherches, loisirs) et les appels sur le telephone portable (en moyenne, une demi-douzaine d'appels emis et reçus quotidiennement). La seule dichotomie majeure se situe au niveau de l'usage des textos, plus rare chez les jeunes Bresiliens, qui en envoient en moyenne un par jour, et extrêmement frequent dans le groupe français, dont la majorite envoient et reçoivent entre 20 et 100 SMS par jour. Cette difference d'usage favorise autant qu'elle revèle une communication intense des adolescents français centree sur le groupe de pairs. Diverses etudes montrent que ces derniers ont une connectivite particulièrement haute, $y$ compris en comparaison avec les jeunes Europeens, Nord-americains et Asiatiques, qui plebiscitent egalement les SMS pour communiquer entre eux (DOCOMO, 2009; Energy BBDO, 2006; Lenhart, Ling, Campbell, \& Purcell, 2010). 
À la question ouverte " qui sont les trois personnes avec qui tu communiques le plus sur ton telephone portable? ", sans distinction entre appels et textos, ni entre communications emises ou reçues, la majorite des adolescents ont cite " ma mère " et « un(e) ami(e) " parmi ces trois personnes. Dans la figure 6, afin de mettre en valeur l'importance relative de ces differentes communications, une valeur de trois points a ete attribuee au contact cite comme le plus frequent, deux points pour le contact cite en deuxième position, et un point pour le contact cite en troisième position. Le tableau montre que si la mère apparaît globalement comme le contact le plus frequent (premier contact des jeunes Cariocas et deuxième contact des jeunes Franciliens), les communications avec le groupe des pairs surpassent largement celles avec la mère chez les jeunes Franciliens ${ }^{14}$.

Figure 6 : Contacts les plus fréquents sur le téléphone portable (points)*

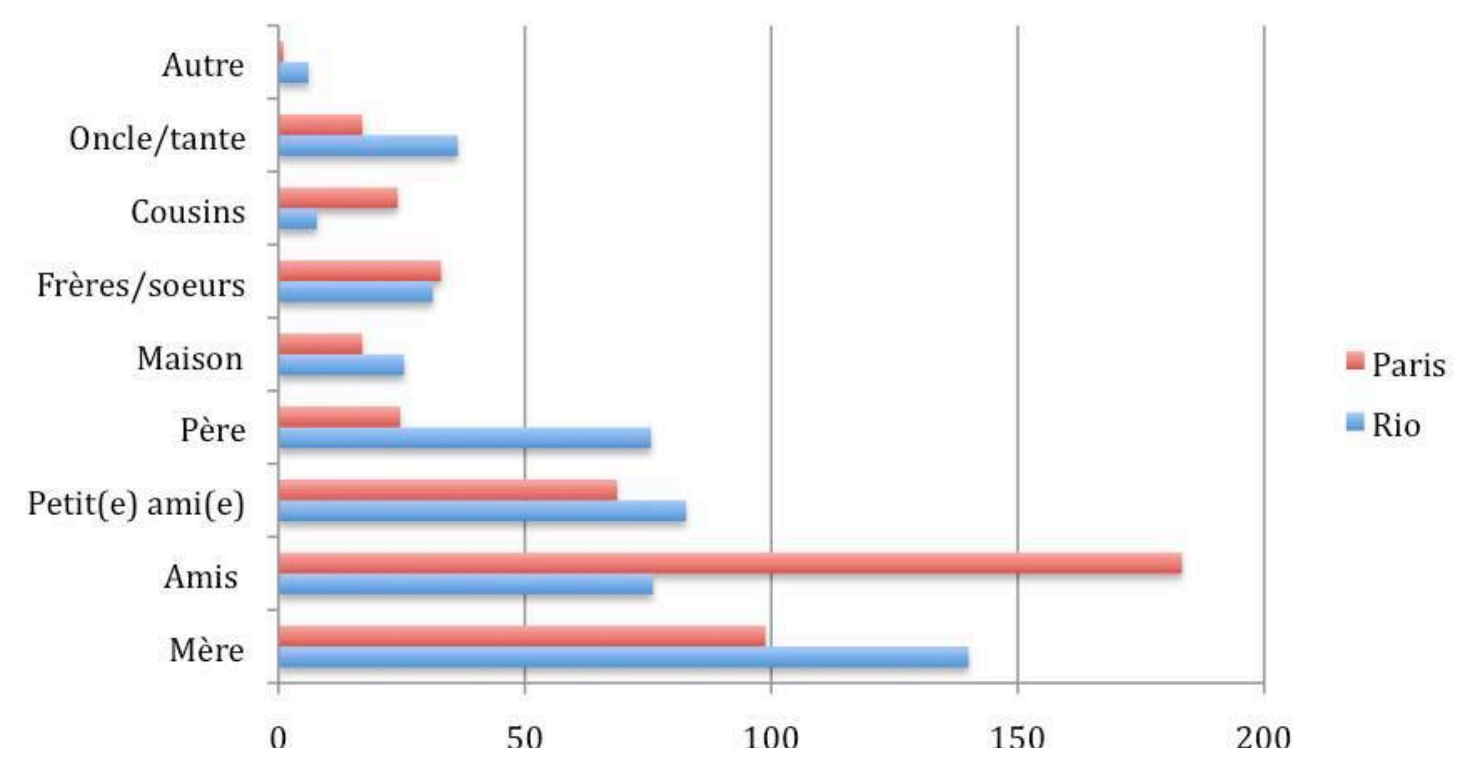

* Premier contact $=3$ points ; deuxième contact $=2$ points ; troisième contact $=$ un point.

$\mathrm{Si}$ l'on opère des regroupements entre les contacts cites, deux clivages principaux apparaissent : I'âge et les liens familiaux. En effet, alors que les contacts avec les membres de la famille et avec les amis sont relativement equilibres dans le groupe francilien, la figure 7 montre bien la preponderance de la famille dans les communications telephoniques du groupe carioca $^{15}$. De plus, quand ces contacts sont regroupes par generation, on remarque que les Cariocas ont legèrement plus de contacts avec leurs aînes, tandis que les Franciliens ont très nettement plus de contacts avec leur groupe de pairs (figure 8).

\footnotetext{
14 Même en considérant que la réponse «ma mère » ne peut être citée qu'une seule fois, tandis qu' « un(e) ami(e) » peut apparaître jusqu'à trois fois, l'ampleur de l'écart entre les deux réponses permet d'affirmer que « un(e) ami(e) » est davantage cité en première position que «ma mère » par les jeunes Franciliens. Dans le cas d'une réponse comme : 1) ma mère 2) un ami 3) un ami, le poids des réponses «ma mère » et «un(e) ami(e) » serait égal, avec 3 points pour chacune.

15 Dans ce regroupement, les réponses «petit(e) ami(e)»n'ont été intégrées dans aucun des deux groupes, car les relations amoureuses peuvent relever autant de la socialisation entre pairs que de l'importance de la construction d'une famille.
} 
Figure 7 : Contacts les plus fréquents sur le téléphone portable : famille/amis (points)

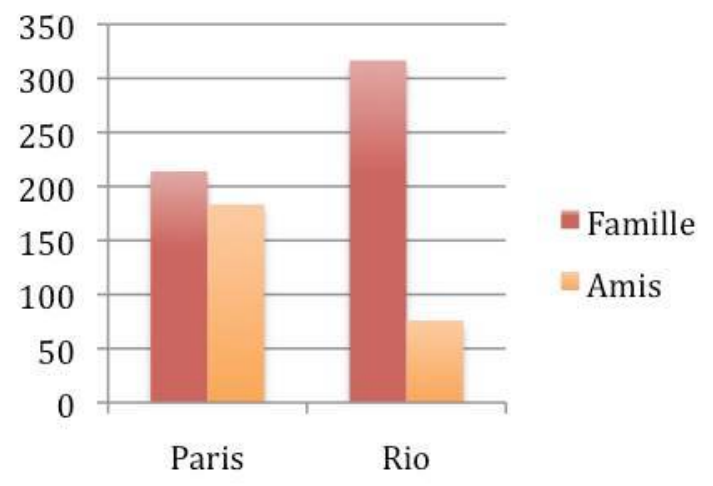

Figure 8 : Contacts les plus fréquents sur le téléphone portable : génération (points)

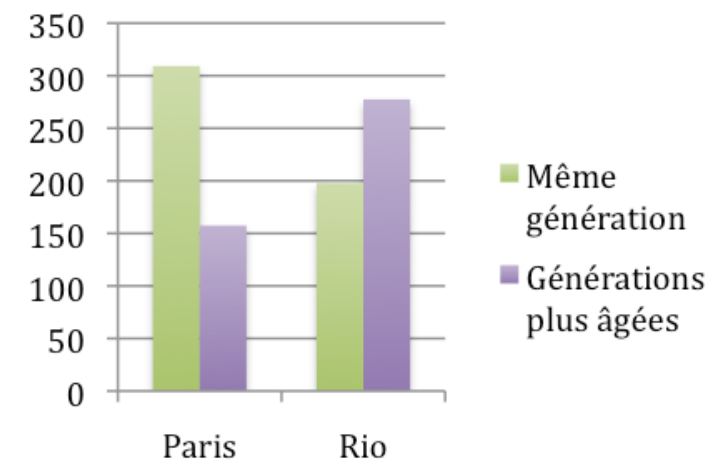

Cette difference dans les sociabilites numeriques reflète les differentes modalites culturelles de la socialisation et differents "repertoires culturels " (Lamont \& Thevenot, 2000; Prost, 1968) en France et au Bresil. En effet, la famille est une institution très forte au Bresil, où elle a assume durant des siècles le rôle principal dans l'organisation de la vie economique, sociale et spatiale (Buarque de Holanda, 1984; Freyre, 1954; Reis, 1998; Samara, 1997). En revanche en France, la tradition ancienne d'un État fort et d'un reseau administratif dense, se reflète dans un système scolaire qui regit le quotidien des enfants et adolescents et a favorise la sociabilite generationnelle (Galland, 2009; Prost, 1968; Vallet \& Thelot, 2000; Van de Velde, 2008).

On sait que les telecommunications, à l'ère du numerique comme à celle du telephone fixe, s'effectuent avant tout avec les personnes proches que l'ont voit le plus souvent (Christakis \& Fowler, 2009; Smoreda \& Licoppe, 1999). Dans cette optique, la telecommunication n'est pas une alternative à ou une substitution de la communication en copresence, mais son complement ou son prolongement. Alors qu'en France les adolescents passent la majeure partie de leur temps au lycee avec d'autres adolescents, les jeunes Bresiliens ne passent que trois ou quatre heures par jour dans les lycees publics. Une partie importante de leurs journees se passe donc à la maison, où ils cohabitent souvent avec leurs grands-parents, oncles et tantes ou neveux ou nièces. Cependant, les pratiques numeriques ne viennent pas uniquement refleter des pratiques de socialisation, elles les transforment egalement. Ainsi, 
le groupe de pairs soude des Franciliens gagne en autonomie et en mobilite, tandis que la solidarite familiale des Cariocas devient plus compatible avec les loisirs adolescents.

\section{Une sociabilité numérique avec le groupe de pairs qui favorise les rencontres en co- présence}

Les communications numeriques ne servent pas uniquement à maintenir le lien avec le groupe de pairs à distance, mais dans une large mesure à organiser la co-presence (a). En effet, l'importance accordee par les lyceens aux petits gains de temps lies à l'utilisation de technologies qui leur en font aussi perdre beaucoup revèle la pertinence du temps de la sociabilite pour cette classe d'âge (b).

\section{Organiser la coprésence}

À un âge où ils ne sont plus surveilles, mais encore encadres, les adolescents trouvent dans la telephonie mobile un outil facilitateur de sorties de groupe. La possibilite à la fois de communiquer tout au long de la journee grâce au telephone portable, et de differer la lecture ou l'envoi des textos aux moments de pause entre les cours suscite de nombreux echanges pour organiser les rencontres à la sortie du lycee. Il ne s'agit plus de traîner, mais de prevoir de faire quelque chose, comme l'explique Sarah :

J'aime bien quand c'est préparé, j'aime pas aller à Châtelet pour rien faire. J'aime bien sortir, on va au parc de la Courneuve quand il fait beau... Quand je vais à Paris c'est pour aller au musée, faire quelque chose de précis. J'aime pas traîner. [Sarah, 16 ans, lycée Paris Nord]

En outre, les nombreux "Tu sors à quelle heure? " echanges quotidiennement ne permettent pas seulement aux elèves de differentes classes du même etablissement de se retrouver, mais facilitent egalement les rencontres avec des amis et cousins scolarises dans des etablissements du voisinage. En ce sens, les liens tisses au collège ne se maintiennent pas seulement sur les sites de reseautage, mais egalement par des rencontres regulières. Les sorties s'organisent generalement les mercredis et samedis après-midi et, s'il y en a d'autres, les jours de semaines où les cours finissent avant $16 \mathrm{~h}$. II s'agit tout d'abord de trouver un lieu et un motif de reunion : organiser un match de foot, tester un jeu video chez Untel dont les parents ne rentrent qu'à 19h, accompagner Unetelle à Châtelet s'acheter un survêtement pour la danse hip-hop, aller à la mediathèque reviser le prochain contrôle de maths... Ces sorties sont parfois suscitees par la publicite ciblee que les operateurs de telephonie mobile envoient par SMS aux adolescents dans le cadre d'operations de marketing organisees à proximite de chez eux : menus à prix reduits pour l'ouverture d'un nouveau KFC, evenement Nike avec cadeaux publicitaires de la marque, rencontre à la FNAC avec un groupe pour le lancement de leur dernier album, entre autres.

Selon l'activite projetee, le lyceen contacte alors un ami proche par texto, ou tout un groupe par un texto collectif. Les reponses arrivent dans l'heure et le rendez-vous est pris. La dernière etape est de prevenir les parents qui, joignables, et rassures que leur enfant le soit, autorisent la plupart des sorties dans certaines limites d'horaires. Lucas explique :

Quels sont tes trois derniers appels emis ? 
(...) Ma mère, les trois. Elle me laisse faire tout ça mais il faut que je la prévienne où je suis. Elle marche à la confiance quoi. Enfin, je peux pas aller à Paris à $21 \mathrm{~h}$, mais si c'est après les cours, je vais voir un pote et on va rester ensemble jusqu'à 18h30, ça va (...)

T'as combien de contacts telephoniques avec ta mère par jour?

Trois. Après les cours, c'est tout. [Lucas, 15 ans, lycée Paris Sud]

La posture de la mère de Lucas est la plus couramment observee, ce qui permet d'affirmer que dans la majorite des cas, le fait d'être joignable sur leur portable favorise la mobilite des lyceens. Ces derniers precisent d'ailleurs que même avec des parents permissifs, s'il leur fallait rentrer chez eux pour demander l'autorisation de sortie ils ne ressortiraient pas. Neanmoins, dans certains cas, le fait que l'elève possède un telephone portable ne semble pas favoriser l'independance affective des parents, comme pour Nadège, dont la mère telephone constamment et sans motif precis, ce qui ne l'empêche pas de sortir regulièrement avec sa sœur, ses amies et ses cousines après les cours :

Les trois derniers appels émis et reçus c'était quoi ?

Moi j'en ai pas. Ah si ma mère, toujours pour la même chose, pour demander je suis où [interference de portable] ah en plus c'est elle ! Allô ? Oui... Je suis avec une dame en fait je fais un sondage sur les technologies je suis au lycee16 (...) [elle raccroche]

Ta mère t'appelle pour vérifier où tu es?

Ou si non elle me dit... Non c'est tout. Je sais pas pourquoi. Elle appelle pour voir je suis où, pour me dire si je vais quelque part, après je dis oui ou non... et quand je suis quelque part elle appelle pour me dire euh... En fait elle même elle sait pas pourquoi elle appelle, donc moi je vais pas savoir! Elle appelle elle dit " t'es où ? " je lui dis, elle dit « ah, ok » et elle raccroche. [Nadège, 16 ans, lycee Sud Paris]

Par contraste, le telephone portable est peu utilise dans le groupe carioca pour demander des autorisations de sorties ou rassurer les parents. En effet la liberte de mouvement est acquise assez tôt, puisque beaucoup d'enfants jouent ensemble dans les rues de la favela à proximite de chez eux, où ils sont en principe sous le regard de la communaute d'adultes, amis ou voisins de leurs parents. Les adolescents utilisent le texto au moment de retrouver un ami, pour lui demander s'il est dejà arrive par exemple, mais pas pour organiser la rencontre. Ceci s'effectue davantage sur Orkut $^{17}$, d'une manière plus aleatoire puisque le site n'est pas toujours consulte quotidiennement :

E os recados que você manda no Orkut, é mais sobre o que?

Só falar "Fernanda, vai hoje pra não sei aonde ? ", aí eu falo " eu vou », " então tá ».

Mas você vai ver só a semana seguinte, você fica duas semanas sem ver.

\footnotetext{
${ }^{16}$ L'entretien avait lieu en milieu d'après-midi, durant les horaires de cours de Nadège.

${ }^{17}$ Orkut est un site de réseautage social lancé par Google en 2004 et qui a remporté très tôt l'adhésion massive de la population brésilienne. Au moment de la recherche, $94 \%$ des élèves brésiliens interrogés utilisaient Orkut, et seuls $2 \%$ Facebook. Actuellement, une migration des utilisateurs brésiliens d'Orkut a commencé vers Facebook.
} 
É. Minha amiga " te mandei recado e você não respondeu ", " poxa, foi mal, porque eu não estava tendo tempo $»^{18}$. [Fernanda, 18 ans, lycee Rio Ouest]

\section{Gagner du temps, pour le passer ensemble}

En France, les NTIC entrent egalement en jeu dans une manière d'organiser la copresence qui vise à faire " gagner " du temps en evitant au maximum les temps d'attente, notamment dans l'organisation des trajets avec les amis :

Tu envoies combien de textos par jour?

Une trentaine. Dès le matin je textote ma copine pour savoir où on se rejoint où pour le bus.

Pourquoi vous ne prenez pas rendez-vous la veille?

Parce que moi je prends le bus avant elle, donc dès que je monte dans le bus, je la previens pour qu'elle descende, pour pas qu'elle le loupe. Soit on dit « t'es où ? " (...)

Quels sont tes trois derniers textos reçus et envoyés?

Ma copine, Saba, elle disait " vous êtes où je suis devant le lycee ? ", à 13h11, je lui ai dit " on arrive » (...) Après l'autre copine qui est dans ma classe, elle m'a dit " je suis là » parce que je lui ai dit " t'es où ? " pour le bus, c'etait à $13 \mathrm{~h}$, je venais de prendre le bus en bas de chez moi. Elle me dit " navette ou bus ? " à $12 \mathrm{~h} 58$, parce que des fois on prend la navette et des fois le bus. Je lui ai dit " bus ». [Ombeline, 16 ans, lycee Paris Nord]

Dans la même logique, il n'est pas rare que le premier elève à apprendre l'absence d'un professeur dans la journee envoie un SMS collectif à toute la classe pour annoncer la bonne nouvelle, mais aussi pour que personne n'attende devant la salle de cours. L'acceleration du temps en lien avec les NTIC est une assomption recurrente dans la sociologie contemporaine (Bauman, 2005; Rosa, 2005). Ces dernières encouragent l'accumulation des tâches et la permeabilite des temps des demandes professionnelles et familiales chez les adultes, en particulier chez les femmes (Wajcman, 2008). Cependant, on observe ici que l'usage des NTIC dans une logique de rentabilisation du temps n'est pas seulement le fait d'adultes surmenes, mais egalement une pratique habituelle des lyceens du groupe français, population qui beneficie pourtant d'un temps libre plus important, partage entre la sociabilite, les loisirs et l'ennui.

II peut en effet sembler paradoxal que ces mêmes technologies à la fois occupent beaucoup de temps et soient utilisees pour gagner du temps dans l'organisation des mobilites. Cela revèle en realite la valeur attribuee à certaines occupations implicitement comparees à d'autres. Ainsi, le temps le plus souvent considere comme perdu est celui des transports et de l'attente, qui perdent leur sens face aux technologies qui relativisent les distances par un accès instantane aux personnes, informations et loisirs. En revanche, le temps de la socialisation n'est jamais cite comme du temps perdu - ce qui explique en outre que les

${ }^{18}$ Et les messages que tu envoies sur Orkut, c'est sur quoi en général ?

C'est juste pour dire « Fernanda, tu vas je-sais-pas-où aujourd'hui ? », je dis « oui », elle dit « ok ».

Mais tu ne vas voir le message que la semaine suivante puisque tu passes deux semaines sans regarder.

Et oui. Mon amie me dit «je t'ai envoyé un message, tu m’as pas répondu », «zut, désolée, c'est parce que j'avais pas le temps » 
elèves s'efforcent au maximum de transformer le temps de transports en temps de socialisation en organisant leur mobilite avec leurs amis. Pour certains, la question du temps de socialisation hors de l'ecole apparaît même comme une priorite qui devance les questions du confort materiel de la famille, question pourtant très presente dans les preoccupations des enquêtes, et ce y compris chez les lyceens bresiliens dont les journees de cours sont pourtant bien moins chargees, mais qui les cumulent souvent avec un stage, des cours prives ou un emploi :

Você tem três pedidos pra melhorar seu cotidiano, [quais são] ?

Tipo, mais tempo. Eu acho que eu tenho muito pouco tempo para aproveitar. Eu as 10 horas vou pra escola, chego em casa as 7 horas. Não tenho nenhum tempo. Tenho que ficar em casa só vendo televisão com meu irmão (...). Se eu estudasse de manhã eu teria muito tempo. Eu pediria mais tempo, pediria descanso pra minha mãe e um emprego pra mim.

Se você tivesse mais tempo você ia fazer o que?

Pra ficar com os amigos ${ }^{19}$. [Anilton, 16 ans, Rio Sud]

Si tu pouvais faire trois vœux, qu'est-ce que ça serait?

Avoir moins de cours, j'ai cours tout le temps, presque tout le temps $8 h-5 h 30$ (...).

Si t'avais que $20 \mathrm{~h}$ de cours par semaine, tu ferais quoi en plus?

Je ferais plus de danse dejà, et je pense que je verrais plus mes amis en dehors des cours. [Cynthia, 15 ans, Paris Nord]

On observe donc que la sociabilite, en particulier avec le groupe de pairs, est un des principaux critères de pertinence de l'espace (comme le montre le temoignage de Fabiano sur le manque d'espace public de socialisation dans son quartier de classe moyenne) et du temps (comme en temoignent Anilton et Cynthia ci-dessus). Ainsi, les NTIC permettent de reintroduire de la sociabilite dans les espaces et dans les moments qui en sont depourvus soit par la co-presence virtuelle comme cela a ete montre dans plusieurs etudes (Cardon, Smoreda, \& Beaudouin, 2005; Metton, 2010; Singly \& Martin, 2000), soit par l'organisation de la co-presence physique. La mobilite favorisee par les usages des NTIC est egalement au cœur des communications avec la famille, où elle repond à une logique toute autre.

\section{Des communications numériques pour concilier la solidarité familiale et la mobilité spatiale}

Le fait que parents et adolescents soient joignables favorise la mobilite de ces derniers dans une logique d'autorite ou de responsabilite parentale (demander l'autorisation d'aller à tel endroit, prevenir de ses deplacements), mais egalement dans une logique de solidarite

\footnotetext{
19 Tu peux faire 3 vœux pour améliorer ton quotidien, [quels sont tes vœux]?

Genre, plus de temps. Je trouve que j'ai très peu de temps pour moi. à $10 \mathrm{~h}$ je vais à l'école, le soir j'arrive à 7h. J'ai aucun temps. Il faut que je reste à la maison à regarder la télé avec mon frère (...). Si j'avais cours le matin j'aurais beaucoup plus de temps. Je demanderais plus de temps, que ma mère puisse se reposer, et un emploi pour moi.

Si tu avais plus de temps tu ferais quoi?

Je le passerais avec mes amis. [Anilton, 16 ans, Rio Sud]
} 
familiale, tout particulièrement chez les Cariocas. Celle-ci s'observe d'une part à travers des petits services et deplacements quotidiens (a) et d'autre part à travers des deplacements occasionnels vers la famille plus eloignee (b).

\section{Être disponible pour sa famille sans être bloqué à la maison : une contradiction résolue par le téléphone portable}

L'importance de la famille au Bresil a ete soulignee tout au long du XXe siècle, notamment par les classiques de la pensee sociale bresilienne qui font remonter son origine au rôle central de la famille dans la fondation du Bresil dès l'epoque coloniale et tout au long de l'empire, dans un territoire trop vaste pour être structure par des institutions etatiques et dont l'organisation economique etait centree sur la monoculture d'exportation des grandes fazendas patriarcales (Buarque de Holanda, 1984; Freyre, 1954; Prado Júnior, 1942). Cette interpretation a ete critiquee comme etant une generalisation du modèle de la fazenda du Nordeste, et les historiens et sociologues de la famille ont montre l'importance dans les milieux populaires d'autres modèles familiaux, matrifocaux ou matricentres, et de la solidarite familiale non liee au patrimoine (Peixoto, 2005; Samara, 1997).

Comme je l'ai expose plus haut, les deux ou trois communications moyennes emises et reçues quotidiennement par les jeunes Cariocas sont echangees principalement avec leur famille, en particulier les membres des generations plus âgees. Le plus souvent, les appels visent à solliciter l'aide d'un des membres de la famille. Ces coups de main ou depannages donnent lieu à des deplacements entre les maisons des differents membres de la famille au sein de la favela. En dehors de leurs 3 ou 4 heures de cours de la journee, les adolescents sont par exemple sollicites par leur père, maçon ou electricien, qui appelle son fils pour qu'il vienne l'aider (et apprendre) sur un chantier, ou leur mère quand elle a fini les courses au supermarche en bas de la favela, pour venir chercher les sacs et les remonter jusqu'à la maison à travers les rues en pente raide du morro. Ou encore la grand-mère, qui vit à quelques maisons de chez eux, appelle son petit-fils pour verifier les branchements de la television qui ne fonctionne plus. II se peut que leur mère ou leur tante, qui garde les enfants en bas âge de leur propre sœur leur demande de venir les surveiller une demi-heure, le temps qu'elle apporte le repas qu'elle a prepare pour sa mère, ou qu'elle livre le paquet de linge qu'elle a repasse à sa cliente.

Cette solidarite familiale existe aussi en France, où les adolescents peuvent être contactes sur leur telephone portable pour passer chercher leur petit frère ou petite sœur à la sortie de l'ecole. Cependant, l'adolescent français ne sera generalement sollicite que pour lui demander de faire quelque chose de peu contraignant : acheter le pain sur le chemin de la maison, mais rarement ressortir acheter le pain ; chercher le petit frère à $16 \mathrm{~h} 30$ si lui-même sort du lycee à $16 \mathrm{~h}$, mais plus difficilement s'il sort à $15 \mathrm{~h}$, pour ne pas bloquer son aprèsmidi ou le faire ressortir. En comparaison, les demandes familiales envers les adolescents bresiliens sont plus nombreuses et plus contraignantes, mais ces derniers considèrent leur disponibilite envers la famille comme normale. En raison d'un certain devoir moral de solidarite familiale au quotidien (Peixoto, 2005; Samara, 1997), la telephonie mobile donne aux adolescents une plus grande liberte de mouvement au sein de la favela puisque quitter la maison ne s'apparente pas, quand on est joignable, à nier egoïstement sa disponibilite. Quand la telecommunication n'est pas possible, comme entre Leandro et sa grand-mère, qui n'a aucun telephone, la mobilite spatiale de l'adolescent doit s'y ajuster. Leandro raconte 
qu'il n'aime pas rester chez lui et prefère être dehors, dans les rues de la favela. Mais aux horaires où sa mère travaille, il reste sur la petite place où habite sa grand-mère pour être à portee de voix, ou bien passe au moins toutes les heures voir si elle n'a besoin de rien. Ce n'est pas par peur de l'accident puisque sa grand-mère est entouree de voisins amicaux qui peuvent lui telephoner en cas d'urgence. II s'agit bien plus de montrer à un membre de la famille qu'on ne le laisse pas seul, et qu'on est à disposition pour de petites attentions.

\section{Solliciter la famille "éloignée " pour quitter occasionnellement le lieu de résidence : l'opportunité d'un autre contexte de socialisation}

La solidarite familiale se manifeste egalement de la part des membres de la famille qui n'habitent pas la favela pour permettre à l'adolescent de sortir de la favela le week-end. Cette pratique recouvre plusieurs fonctions. D'une part, il s'agit de developper et maintenir une proximite avec des oncles, tantes ou grands-parents en leur rendant visite, puisqu'on ne les côtoie pas au quotidien. De plus, cela permet à l'adolescent de se faire des amis dans un autre quartier, amis souvent consideres par les parents voire par les adolescents eux-mêmes comme de meilleures frequentations que les amis de la favela, si c'est un quartier de classe moyenne. Enfin, il s'agit pour certains parents d'eviter que leurs enfants - surtout les filles aillent au baile funk, fête qui reunit des milliers de jeunes au son du funk carioca les nuits du vendredi et du samedi, et que la plupart des parents considèrent comme un lieu de perdition en raison des paroles crues et des danses suggestives qui caracterisent cette musique, et de la drogue qui circule dans la soiree. À cela s'ajoutent les risques de balles perdues la nuit dans les rues de la favela, qui representent une raison de plus pour ne pas y être le samedi soir. La mère de Fernanda a ete explicite à ce sujet :

Você tem quantos amigos no seu Orkut ?

Mais de 600, mas tem 100 que eu não conheço, que são amigos dos meus amigos. Mas o resto, e daqui e de Nilópolis, porque eu ficava muito lá em Nilópolis. Porque minha tia morava lá, e minha mãe falava "de segunda a sexta fica aqui, sábado e domingo tu vai pra lá, porque lá não tem nada" (baile funk, drogas, tiroteio)

Demora quanto tempo para ir pra lá?

Pego duas conduções, duas horas.

Você gostava?

Gostava, tem clubes, tem um monte de coisas que e mais social, mais organizado. Não tem... Você pode entrar na hora que você quiser que você não vê uma arma, não vê uma pessoa fumando, uma pessoa cheirando." ${ }^{20}$ [Fernanda, 18 ans, lycee Rio Ouest]

\footnotetext{
${ }^{20}$ Tu as combien d'amis sur Orkut?

Plus de 600, mais y'en a 100 que je ne connais pas, c'est des amis de mes amis. Mais le reste c'est des gens d'ici et de Nilópolis, parce que j'allais souvent à Nilópolis. Parce que ma tante habitait là-bas et ma mère disait « du lundi au vendredi tu restes ici, le samedi et le dimanche tu vas là-bas, parce que là-bas y’a rien » (pas de baile funk ni de drogue ou de coups de feu)

Tu mets combien de temps pour y aller?

Je prends deux bus. Deux heures.

Tu aimais bien?
} 
Comme tout le monde n'a pas de famille dans des quartiers à la fois accessibles et surs, il est très frequent qu'une mère employee comme domestique auprès de la même patronne depuis longtemps demande à cette dernière si elle accepterait d'être la marraine ${ }^{21}$ de son enfant. Ce glissement de la relation de travail à la relation familiale peut s'operer en raison de la position intermediaire qu'occupe la domestique entre ces deux sphères et en raison du regard paternaliste que portent les employeurs sur leur domestique (Vidal, 2007). En acceptant, la marraine est liee par un devoir d'aide envers son (sa) filleul(e) qui peut se traduire, si elle le remplit, par des dons de vêtements, plus tard d'un ordinateur, et parfois même en lui payant quelques annees de scolarite privee. Quand la relation est bonne entre marraine et filleul(e), il ou elle peut passer le week-end chez elle, comme le fait Tamara, dont la marraine est la patronne de sa mère :

Você vai na Zona Sul?

(...) em Copacabana, minha madrinha ainda mora lá. Então sempre estou lá, praticamente. Quando dá. Durmo lá quando eu não trabalho. Aí quando não tem nada pra fazer aqui em casa ou com minhas amigas, eu pego (o ônibus) e vou para a minha madrinha (...).

No seu Orkut você tem quantos amigos?

600 e pouco. Mas eu só adiciono quem eu conheço mesmo.

600 ! Você conhece eles da onde?

Gente que eu conheci em Niterói, gente que eu conheci em Copacabana, amigos que estudaram comigo em várias escolas... assim vai. Amigos que eu conheço, assim, tem Orkut para se comunicar, aí eu falo que tenho ${ }^{22}$. [Tamara, 17 ans, lycee Rio Sud]

Les temoignages de Fernanda et Tamara montrent que les liens crees en copresence lors des deplacements auprès de la famille dans d'autres lieux sont maintenus sur Orkut. Les Franciliens temoignent egalement de contacts sur MSN, sur Skyblog dans leurs annees collège et plus recemment Facebook avec des copains connus durant les vacances passees en province ou à l'etranger dans la famille, sans que cela soit lie pour autant à une volonte explicite de diversifier leur reseau social ou de les eloigner de leur quartier. Le maintien de ces liens via Internet leur apporte toutefois des benefices, comme l'apprentissage d'une langue etrangère. Ainsi, $28 \%$ des lyceens franciliens declarent utiliser une langue autre que le français ou l'anglais sur Internet.

Oui j'aimais bien, il y a des boîtes, il y a plein de choses plus formelles qu'ici, plus organisées. Il n'y a pas... Tu peux rentrer à l'heure que tu veux, tu ne vas pas voir une arme à feu, pas une personne en train de fumer (du cannabis), pas une personne en train de sniffer.

${ }^{21}$ Le soin des enfants étant une activité traditionnellement féminine, les marraines sont davantage citées que les parrains.

22 Tu vas dans la Zona Sul?

(...) à Copacabana, ma marraine y habite toujours. Du coup j'y suis presque tout le temps. Quand je peux. Je dors là-bas quand je ne travaille pas. Et quand y'a rien à faire à la maison ou avec mes copines, je prends le bus et je vais chez ma marraine (...).

Tu as combien d'amis sur Orkut?

600 et quelques. Mais je n'ajoute que ceux que je connais vraiment.

600 ! Tu les connais d'où?

Y'en a que j'ai connus à Niteroi, d'autres à Copacabana, des amis qui étaient avec moi dans plusieurs écoles... etc. Des amis que je connais, comme ça on a Orkut pour communiquer, donc je leur dis que je l'ai. 
On observe donc qu'à partir de visites occasionnelles auprès d'un referent familial de la generation de leurs parents, les adolescents developpent en ligne un reseau social juvenile ancre dans un autre espace physique, qu'ils peuvent retrouver lors de leurs deplacements. La solidarite familiale qui consiste à accueillir le week-end un(e) filleul(e) ou neveu ou nièce ou petit enfant qui habite une favela dans le cas carioca, ou à le faire durant les vacances dans le cas francilien, en s'articulant avec la sociabilite numerique, finit par ancrer l'adolescent dans un espace physique supplementaire à travers les liens sociaux qu'il y a tisses.

\section{Conclusion}

Cet article s'est concentre sur une partie de la sociabilite numerique constituee par les communications pragmatiques, c'est-à-dire qui visent à transmettre une information concrète, auxquelles il faudrait ajouter toutes les communications phatiques ${ }^{23}$ ainsi que les conversations de longue duree s'apparentant à de la copresence virtuelle. Les resultats font apparaître que la joignabilite dans l'espace numerique permet aux adolescents d'orienter leur presence physique vers l'espace où elle est la plus pertinente à un moment donne, pour participer à une activite entre amis ou pour remplir son rôle au sein de la famille. En outre, le lien numerique semble encourager l'exploration de l'espace par les adolescents en agissant comme une longue amarre les rattachant à leurs parents. Loin de se substituer aux rencontres en presence, les communications les favorisent en multipliant les contacts et en facilitant l'organisation des deplacements. Les adolescents gagnent en mobilite spatiale du fait qu'ils sont toujours joignables par leur famille, aux yeux de laquelle ils ne doivent se soustraire ni à l'autorite parentale, ni à la solidarite familiale.

En mettant en relief les similitudes dans les equipements et communications numeriques ainsi que dans l'augmentation de la mobilite urbaine qui en decoule, cette etude transnationale effectuee dans deux metropoles du Nord et du Sud indique qu'une certaine convergence des normes de sociabilite peut être observee auprès d'adolescents habitant des grands pôles urbains mais ne faisant pas partie des classes moyennes et superieures chez qui on observe plus souvent une globalisation des styles de $v^{24}$. Toutefois, les sociabilites numeriques s'inscrivent egalement dans la continuite de sociabilites dominees soit par le groupe de pairs à Paris, soit par la famille à Rio de Janeiro, c'est-à-dire par un groupe constitue avant tout dans l'espace physique et selon des normes culturelles locales.

\section{Bibliographie}

BAUERLEIN M. (2008), The Dumbest Generation. How the Digital Age Stupefies Young Americans and Jeopardizes our Future, Tarcher/Penguin, $272 \mathrm{p}$.

BAUMAN Z. (2005), Liquid Life, Polity, 174p

\footnotetext{
${ }^{23}$ dont le but est le contact en lui-même, et non la transmission d'informations.

${ }^{24}$ Cette étude sur les pratiques numériques des jeunes de milieux populaires ne fait pas apparaître de différence majeure avec celles des jeunes de classes moyennes et élites telles que relatées dans la littérature sociologique, bien qu'une comparaison sociale plus détaillée le ferait certainement.
} 
BEAUDE B. (2011) « De l'importance des lieux reticulaires ", Carnets de Géographes, 2011/2 [Electronic Version]

BECK U. (2007), " La condition cosmopolite et le piège du nationalisme methodologique ", in M. Wieviorka (Ed.), Les Sciences sociales en mutation, Auxerre, Editions Sciences Humaines, pp. 223-236.

BECK U. et BECK-GERNSHEIM E. (2008) « Global Generations and the trap of methodological nationalism for a cosmopolitan turn in the sociology of youth and generation ", European Sociological Review, 25-1, pp. 25-36.

CARDON D., SMOREDA Z. et BEAUDOUIN V. (2005). " Sociabilites et entrelacement des medias ", in P. Moati (ed.) Nouvelles technologies et modes de vie, Paris, l'Aube.

CARTIER,M., COUTANT I., MASCLET, O. et SIBLOT Y. (2008), La France des "petits-moyens". Enquête sur la banlieue pavillonnaire, Paris, La Decouverte, 317p.

CHRISTAKIS N. A. et FOWLER J. H. (2009), Connected. The surprising power of our social networks and how they shape our lives, New-York, Little, Brown and Company, 338p.

CORREA D. (2006), "Estudos sobre Comunicação e Cibercultura no Brasil: Conceitos, Tendências e Clusters ", Razón y Palabra, 53 [Electronic Version].

CREDOC (2009), La Diffusion des technologies de l'information et de la communication dans la société française, 220p.

DELAUNAY-TETEREL H. (2010), "L'affichage public des amities. Le blog au lycee », Ethnologie française, $\mathrm{XL} / 1$, pp.115-122.

DOCOMO G. N. (2009), Children's Use of Mobile Phones - An International Comparison, GSM Association and Mobile Society Research Institute.

DUPUY G. (2007), La Fracture numérique, Paris, Ellipses.

EDMUNDS J. et TURNER B. S. (2005) «Global generations: social change in the twentieth century », The British Journal of Sociology, 56-4, pp.559-577.

ENERGY BBDO (2006), GenWorld. The New Generation of Global Youth, Chicago, Energy BBDO.

EPOCA (2008), « Quem e a nova classe media do Brasil », Epoca, 534, pp.91-101.

FLORIDA R. (2003), The Rise of the Creative Class: And how it's transforming work, leisure, community, \& everyday life, New York, Basic Books, 434p.

FREYRE G. (1954), Casa grande e senzala. Formação da Família Brasileira sob o Regime de Economia Patriarcal, Rio de Janeiro, Jose Olympio.

GALLAND O. (2009), Les jeunes (7e ed.), Paris, La Decouverte, 124p.

HARGITTAI E. (2010) «Digital $\mathrm{Na}(\mathrm{t})$ ives? Variation in Internet Skills and Uses among Members of the 'Net Generation' ", Sociological Inquiry, 80-1, pp.92-113.

HASSENTEUFEL P. (2005), " De la comparaison internationale à la comparaison transnationale. Les deplacements de la construction d'objets comparatifs en matière de politiques publiques ", Revue française de science politique, 55-1, pp.113-132. 
HOLANDA S. B. (1984), Raízes do Brasil, Rio de Janeiro, Jose Olympio.

HOWE N. et STRAUSS W. (1991), Generations. The History of America's Future, 1584 to 2069, Harper Perennial, 538p.

IANNI O. (1998) "As ciências sociais na epoca da globalização ", Revista Brasileira de Ciências Sociais, 13-37, pp.33-41.

JACOBS J. (1989), The Death and Life of Great American Cities, Vintage Books, 458p.

JARRIGEON A. et MENRATH J. (2010), " De la creativite partagee au chahut contemporain. Le telephone mobile au lycee », Ethnologie française, XL-1, pp.109-114.

JOSEPH I. (1998), La ville sans qualités, Paris, L'Aube, 209p.

KJELDGAARD D. et ASKEGAARD S. (2006) " The Glocalization of Youth Culture: The Global Youth Segment as Structures of Common Difference ", Journal of Consumer Research, 2006/33, pp.231-247.

LAMONT, M. et THEVENOT, L. (Eds.). (2000), Rethinking comparative cultural sociology. Repertoires of evaluation in France and the United States, Cambridge/New York, Cambridge university press, $375 p$.

LARDELLIER, P. et BRYON-PORTET, C. (2010) « Ego 2.0. Quelques considerations theoriques sur l'identite et les relations à l'ère des reseaux ", Les Cahiers du Numérique, 1, pp. 13-34.

LENHART A., LING R., CAMPBELL S. et PURCELL, K. (2010), Teens and mobile phones. Text messaging explodes as teens embrace it as the centerpiece of their communication strategies with friends, Washington DC, Pew Internet.

LIVINGSTONE S. et HADDON, L. (2009), EU Kids Online: Final report, London School of Economics (LSE).

MEC (2009), Ensino Médio inovador, MINISTÉRIO DA EDUCAÇÃO.

METTON C. (2010), "L'autonomie relationnelle. SMS, "chat" et messagerie instantanee ", Ethnologie française, $\mathrm{XL} / 1$, pp.101-107.

NICOLACI-DA-COSTA A. M. (2005), "Sociabilidade virtual: separando o joio do trigo ", Psicologia \& Sociedade, 17/2, pp.50-57.

PASQUIER D. (2005), Cultures lycéennes, la tyrannie de la majorité, Paris, Autrement, 180p.

PASSARELLI HAMANN F. (2004), Comunidade orkutiana brasileira: Considerações sobre jovens e Orkut no Brasil, memoire de Master en psychologie, Pontifícia Universidade Católica do Rio de Janeiro.

PEIXOTO C. E. (2005), "Solidariedade familiar intergeracional ", in C. ARAUJO et C. SCALON (Eds.), Gênero, familia e trabalho no Brasil, Rio de Janeiro, FGV, pp. 225-240.

PEWRESEARCHCENTER (2010), Millennials. A Portrait of Generation Next, Pew Research Center, 140p.

PRADO JUNIOR C. (1942), Formação do Brasil contemporâneo: colônia, São Paulo, Martins.

PRENSKY M. (2001), « Digital Natives, Digital Immigrants », On The Horizon, 9/5, pp. 225-240.

PROST A. (1968), Histoire de l'enseignement en France, 1800-1967, Paris: Armand Colin. 
PUTNAM R. (2000), Bowling alone: the collapse and revival of American community, New York: Simon \& Schuster.

RECUERO R. (2006), "Dinâmicas de Redes Sociais no Orkut e Capital Social ", Razón y Palabra, 52, pp.1-15.

REIS E. P. (1998), "Desigualdade e solidariedade: uma releitura do "familismo amoral" do Banfield ", In E. P. Reis (Ed.), Processos e escolhas: estudos de sociologia política, Rio de Janeiro, Contra Capa, pp.111-134.

REZENDE SALES S. et ALVES PARAISO M. (2010), " Escola, Orkut e juventude conectados: falar, exibir, espionar e disciplinar ", Pro-Posições, 21/2, pp.225-242.

RIVIERE C.-A. (2004), " La specificite française de la construction sociologique du concept de sociabilite ", Réseaux, 1/123, pp.207-231.

ROSA, H. (2005), Accélération, Paris, Editions La Decouverte.

SAMARA E. d. M. (1997), "A família no Brasil: história e historiografia ", História Revista, 2/2, pp.07-21.

SASSEN S. (2006), Cities in a World Economy, Thousand Oaks, Pine Forge Press, 269p.

SELWYN N. (2009), "The digital native - myth and reality ", Aslib proceedings : New information perspectives, 61/4, pp.363-379.

SILVA PEREIRA C. d. (2007), " Os wannabees e suas tribos: adolescência e distinção na Internet ॥, Estudos Feministas, 15/2, pp.357-382.

SINGLY F. d. et MARTIN O. (2000), "L'evasion amicale. L'usage du telephone familial par les adolescents ", Réseaux, 103, pp.91-118.

SMOREDA Z. (Ed.). (2007), Entrelacement des pratiques de communication et de loisir, Paris, Hermes-Lavoisier, 463p.

SMOREDA Z. et LICOPPE C. (1999), La téléphonie résidentielle des foyers : réseaux de sociabilité et cycle de vie, 2ème Colloque International sur les Usages et Services des Telecommunications, Bordeaux.

TAPSCOTT D. (2008), Grown Up Digital, McGraw-Hill, 368p.

TNS/SOFRES (2009), Les adolescents, leur téléphone portable et I'Internet mobile.

TWENGE J. M. (2006), Generation Me: Why Today's Young Americans Are More Confident, Assertive, Entitled - And More Miserable Than Ever Before, Free Press, 292p.

UNIVERSAL MCCANN (2007), Anytime, Anyplace: Understanding the connected génération, Universal McCann, 36p.

URRY J. (2000), Sociology beyond societies. Mobilities for the twenty-first century, London, Routledge, 265p.

VALLADARES L. (2006), La favela d'un siècle à l'autre: mythe d'origine, discours scientifiques et représentations virtuelles, Paris, Editions de la MSH, 229p.

VALLET L.-A. et THELOT C. (2000), « La reduction des inegalites sociales devant l'ecole depuis le debut du siècle ", Économie et Statistique, 4/334, pp.3-32. 
VAN DE VELDE C. (2008), Devenir adulte, Sociologie comparée de la jeunesse en Europe, Paris, PUF, 278p.

VIDAL D. (2007), Les bonnes de Rio. Emploi domestique et société démocratique au Brésil, Villeneuve d'Asq, Presses Universitaires du Septentrion, 312p.

WAJCMAN J. (2008), "Life in the fast lane? Towards a sociology of technology and time ", The British Journal of Sociology, 59/1, pp.59-77.

ZIMIC S. (2010), Opening the Bow - Exploring the presumptions about the "Net Generation ", Mid Sweden University, Sundsvall, thèse de doctorat en communication, 109p. 